\title{
D-dimer, CRP, PCT, and IL-6 Levels at Admission to ICU Can Predict In-Hospital Mortality in Patients with COVID-19 Pneumonia
}

\author{
Marija Milenkovic $\mathbb{D}^{1,2}$ Adi Hadzibegovic $\mathbb{D}^{1},{ }^{1}$ Mirjana Kovac, ${ }^{3}$ Bojan Jovanovic $\mathbb{D}$, ${ }^{1,2}$ \\ Jovana Stanisavljevic, ${ }^{1,2}$ Marina Djikic, ${ }^{1}$ Djuro Sijan, ${ }^{2}$ Nebojsa Ladjevic, ${ }^{1,2}$ Ivan Palibrk, ${ }^{1,2}$ \\ Marija Djukanovic, ${ }^{1,2}$ Jelena Velickovic, ${ }^{1,2}$ Sanja Ratkovic, ${ }^{1,2}$ Milica Brajkovic, ${ }^{4}$ \\ Viseslav Popadic $\mathbb{D D}^{4}{ }^{4}$ Slobodan Klasnja, ${ }^{4}$ Borislav Toskovic, ${ }^{2,4}$ Darko Zdravkovic, ${ }^{2,4}$ \\ Bogdan Crnokrak, ${ }^{2,4}$ Olivera Markovic, ${ }^{2,4}$ Jelica Bjekic-Macut, ${ }^{2,4}$ Aleksandra Aleksic, \\ Simona Petricevic, ${ }^{4}$ Lidija Memon, ${ }^{4}$ Ana Milojevic, ${ }^{4}$ and Marija Zdravkovic $\mathbb{D}^{2,4}$ \\ ${ }^{1}$ University Clinical Centre of Serbia, Belgrade, Serbia \\ ${ }^{2}$ Faculty of Medicine, University of Belgrade, Belgrade, Serbia \\ ${ }^{3}$ Blood Transfusion Institute of Serbia, Belgrade, Serbia \\ ${ }^{4}$ University Clinical Hospital Center Bezanijska Kosa, Belgrade, Serbia
}

Correspondence should be addressed to Adi Hadzibegovic; a.hadzibegovic@gmail.com

Received 7 July 2021; Accepted 31 January 2022; Published 28 February 2022

Academic Editor: Md Hasnain

Copyright (c) 2022 Marija Milenkovic et al. This is an open access article distributed under the Creative Commons Attribution License, which permits unrestricted use, distribution, and reproduction in any medium, provided the original work is properly cited.

\begin{abstract}
Introduction. Health care workers have had a challenging task since the COVID-19 outbreak. Prompt and effective predictors of clinical outcomes are crucial to recognize potentially critically ill patients and improve the management of COVID-19 patients. The aim of this study was to identify potential predictors of clinical outcomes in critically ill COVID-19 patients. Methods. The study was designed as a retrospective cohort study, which included 318 patients treated from June 2020 to January 2021 in the Intensive Care Unit (ICU) of the Clinical Hospital Center "Bezanijska Kosa" in Belgrade, Serbia. The verified diagnosis of COVID-19 disease, patients over 18 years of age, and the hospitalization in ICU were the criteria for inclusion in the study. The optimal cutoff value of D-dimer, CRP, IL-6, and PCT for predicting hospital mortality was determined using the ROC curve, while the Kaplan-Meier method and log-rank test were used to assess survival. Results. The study included 318 patients: 219 (68.9\%) were male and 99 (31.1\%) female. The median age of patients was 69 (60-77) years. During the treatment, 195 (61.3\%) patients died, thereof 130 male (66.7\%) and 65 female (33.3\%). 123 (38.7\%) patients were discharged from hospital treatment. The cutoff value of IL-6 for in-hospital death prediction was $74.98 \mathrm{pg} / \mathrm{mL}$ (Sn 69.7\%, Sp 62.7\%); cutoff value of CRP was $81 \mathrm{mg} / \mathrm{L}$ (Sn 60.7\%, Sp 60\%); cutoff value of procalcitonin was $0.56 \mathrm{ng} / \mathrm{mL}$ (Sn $81.1 \%$, Sp 76\%); and cutoff value of Ddimer was $760 \mathrm{ng} / \mathrm{mL}$ FEU (Sn 63.4\%, Sp 57.1\%). IL-6 $\geq 74.98$ pg/mL, CRP $\geq 81 \mathrm{mg} / \mathrm{L}, \mathrm{PCT} \geq 0.56 \mathrm{ng} / \mathrm{mL}$, and D-dimer $\geq 760$ $\mathrm{ng} / \mathrm{mL}$ were statistically significant predictors of in-hospital mortality. Conclusion. IL- $6 \geq 74.98 \mathrm{pg} / \mathrm{mL}, \mathrm{CRP}$ values $\geq 81 \mathrm{mg} / \mathrm{L}$, procalcitonin $\geq 0.56 \mathrm{ng} / \mathrm{mL}$, and D-dimer $\geq 760 \mathrm{ng} / \mathrm{mL}$ could effectively predict in-hospital mortality in COVID-19 patients.
\end{abstract}

\section{Introduction}

In December 2019, SARS-CoV-2 was identified for the first time as a cause of COVID-19 disease by Chinese scientists [1]. However, after more than a year since the pandemic's beginning, we still do not have a complete picture of the disease itself.

Initially, COVID-19 was considered a respiratory disease, with pneumonia being the most common and deadliest complication. However, SARS-CoV-2 has been shown to 
trigger an excessive and uncontrolled immune-hemostasis response that causes many complications, such as thrombosis, tissue damage, ARDS, DIC, and MODS; therefore, not only it is necessary to understand COVID-19 as a respiratory, but also as a potential multisystem disease $[2,3]$.

Studies from the beginning of pandemic estimated overall hospital mortality from COVID-19 are approximately $15 \%$ to $20 \%$, but up to $40 \%$ among patients requiring ICU admission; however, mortality rates vary across age cohorts, from $5 \%$ among patients younger than 40 years to greater than $60 \%$ for patients aged 80 to 89 years [4]. In contrast, a recent study suggested lower mortality due to the presence of appropriate treatment and vaccination [5].

It is well known that thrombosis is an important complication that significantly increases the risk of a deadly outcome. A great number of thrombosis was verified in SARS-COV2-positive patients [6-8]. Even, despite standard thromboprophylaxis doses of LMWH, $31 \%$ of individuals with proven COVID-19 pneumonia developed thrombosis in ICU [9]. Significant changes in coagulation parameters were verified in patients with COVID-19. Concerning the patients, higher values of $\mathrm{D}$-dimer were observed in persons requiring treatment in ICU [10]. Consequently, higher Ddimer values were associated with severe clinical presentation of COVID-19 disease, as mentioned above [11-14].

Considering all of this, D-dimer and other inflammatory parameters such as IL-6, CRP, and PCT might be used to predict mortality. Predictors of mortality among laboratory parameters are important as they can reflect possible mechanisms of disease progression and give important information on potentially useful therapeutic modalities [15]. Adequate and precise predictors are crucial, especially in the pandemics era.

The aim of this study was to identify potential biochemical predictors of in-hospital mortality among COVID-19 patients and to determine their predictive cutoff values.

\section{Methods}

2.1. Study Design and Participants. The study was designed as a retrospective cohort study, which included 318 patients treated from June 2020 to January 2021 in the Clinical Hospital Center "Bezanijska Kosa" ICU in Belgrade. The criteria for inclusion in the study were the verified diagnosis of COVID19 disease, patients over 18 years of age, and hospitalization in the ICU. The criteria for excluding patients from the study were incomplete data, the patient's stay in the ICU for some other reasons not due to complications of COVID-19 (e.g., postoperative treatment of patients), and transfer of patients to other medical institutions for COVID-19 treatment.

2.2. Definitions, Diagnosis, and Outcomes. COVID-19 diagnosis was made based on the clinical symptoms and signs of the disease, with/without a positive radiological finding (X-ray, CT), and a positive result of the nasopharyngeal swab SARS-CoV-2, detected by the RT-PCR method. The main clinical criteria for Respiratory ICU admission was radiographic or CT scan severity score progression, peripheral oxygen saturation (Sp02) below 93\% despite maximal conventional supportive oxygen therapy (up to $15 \mathrm{~L} / \mathrm{min}$ through a nasal cannula, conventional oxygen, or nonrebreather mask), laboratory test results, mainly an increase of inflammatory parameters after repeated controls, and arterial blood gas test. Critically ill patients on invasive, noninvasive ventilation and high flow oxygen therapy with moderate and severe ARDS were selected for the study according to the Berlin definition of ARDS [16]. The primary outcome of interest was in-hospital mortality and was stratified as deceased or discharged from the hospital. Survivors refer to participants who were discharged from the hospital, and no survivors refer to deceased participants. All patients were followed until their outcomes.

2.3. Treatment. During the hospitalization, patients were treated according to the adjusted National protocol of the Republic of Serbia to treat COVID-19 infection [17]. Antiviral agents (favipiravir, remdesivir) were used 5-7 days from symptom onset in patients on supportive oxygen therapy and with radiographically verified severe bilateral pneumonia. Corticosteroids (prednisone $0.5 \mathrm{mg} / \mathrm{kg}$ in two doses, methylprednisolone $1-2 \mathrm{mg} / \mathrm{kg}$, and dexamethasone $6 \mathrm{mg} /$ day) were used in patients with moderate to severe clinical image with signs of clinical deterioration or in patients with incipient or developed ARDS. Anticoagulant therapy was used in the standard prophylactic dose of LMWH for patients with multiple risk factors and conventional oxygen therapy. According to the anti-Xa levels, therapeutical doses were used for patients in the ICU requiring mechanical ventilation or high-flow oxygen therapy, those on long-term anticoagulant therapy, or those with suspectable or confirmed thrombosis. Antibiotics were used empirically or according to the antibiogram. The main criteria for tocilizumab administration were an increase in IL-6 values above $40 \mathrm{pg} / \mathrm{mL}$ and CRP values above $50 \mathrm{mg} / \mathrm{L}$ or a threefold increase during the last $48 \mathrm{~h}$ in patients with clinical worsening with more than $25 \mathrm{resp} / \mathrm{min}$, saturation below $93 \%$, and partial presure of oxygen below $8.66 \mathrm{kPa}$ without supportive oxygen therapy. Convalescent plasma was used in patients with rapid worsening, positive PCR test for SARS-CoV-2 virus, in the first two weeks from symptom onset. The indication was established according to the specific scoring system with different variables, including the patient's clinical status, a form of the disease, time from symptom onset, respiratory status, radiographic findings, comorbidities, and applied therapy. Inotropic agents, noradrenaline, dobutamine, vasopressin, and adrenaline were used in a standard dosage.

2.4. Data Collection. The necessary data were obtained from the health information system of the Clinical Hospital Center "Bezanijska Kosa" (Heliant, v7.3, r48602). The data includes demographic data (age, gender), laboratory values (IL-6, CRP, PCT, ferritin, D-dimer, lymphocytes, thrombocytes, PT, aPTT, and fibrinogen), and the outcome of the treatment. Past medical history (hypertension, diabetes mellitus, COPD, coronary heart disease, obesity, heart failure, cardiomyopathy, and chronic kidney disease) was obtained from participants' medical documentations and was filed in 
the health information system. Clinical and laboratory parameters were followed upon admission to the hospital and ICU, with specific parameters followed during hospitalization.

2.5. Statistical Analysis. Descriptive statistics methods were used to process and present the results. Continuous variables were presented as the median and IQR and as the frequency (\%) for categorical variables. The Mann-Whitney $U$ test and Pearson's chi-square test were used to compare the data. The optimal cutoff value of D-dimer, CRP, IL-6, and PCT for predicting hospital mortality was determined using the ROC curve, while the Kaplan-Meier method and log-rank test were used to assess survival. A value of $p<0.05$ was considered statistically significant.

\section{Results}

The study included 318 patients, 219 (68.9\%) male and 99 (31.1\%) female. The median age of patients was 69 (60-77) years. During the treatment, $195(61.3 \%)$ patients died: thereof 130 were male $(66.7 \%)$, and 65 were female (33.3\%). 123 (38.7\%) patients were discharged from the treatment. Age, gender, comorbidities, laboratory parameters, and CT score of patients are shown in Table 1.

$C$-indices for IL-6, CRP, PCT, and D-dimer are presented in Table 2. PCT has the highest $C$-index (0.77) to predict in-hospital mortality in COVID-19 patients.

Cutoff values of the analyzed parameters were obtained using the ROC curve. The cutoff value of IL- 6 for in-hospital death prediction was $74.98 \mathrm{pg} / \mathrm{mL}$ (Sn 69.7\%, Sp 62.7\%); cutoff value of CRP was $81 \mathrm{mg} / \mathrm{L}$ (Sn 60.7\%, Sp 60\%); cutoff value of PCT was $0.56 \mathrm{ng} / \mathrm{mL}$ (Sn $81.1 \%$, Sp 76\%); and cutoff value of D-dimer was $760 \mathrm{ng} / \mathrm{mL}$ FEU (Sn 63.4\%, Sp 57.1\%). ROC curves are presented in Figure 1.

Using the Kaplan-Meier survival curve and log-rank test, it was shown that IL-6 higher or equal to $74.98 \mathrm{pg} / \mathrm{mL}$ was a statistically significant predictor of in-hospital mortality $(p=0.04)$. In addition, CRP values higher or equal than CRP $81 \mathrm{mg} / \mathrm{L}$, PCT higher or equal than $0.56 \mathrm{ng} / \mathrm{mL}$, and D-dimer higher or equal than $760 \mathrm{ng} / \mathrm{mL}$ FEU represent significant predictors of in-hospital mortality (CRP, $p=0.02$; PCT, $p<0.001$; and D-dimer, $p=0.04$ ) (Figure 2).

\section{Discussion}

First, our National protocol is mainly following the WHO treatment guidelines [18]. However, we would like to address a few differences between our National protocol and the WHO treatment guidelines. According to our National protocol, the main difference is the usage of favipiravir and remdesivir. The use of systemic corticosteroids, monoclonal antibodies, and IL-6 receptor blockers was in accordance with the WHO treatment guidelines. This slight discordance between protocols should not affect the discussion of our results with results in the literature.

The study indicated significant disorders of laboratory parameters in patients with COVID-19 treated in ICU. Elevated levels of IL-6, CRP, PCT, D-dimer, and lower serum albumin levels were detected in subjects with fatal disease outcomes during treatment. Significantly higher in-hospital mortality was observed in individuals whose IL-6 values were equal to or higher than $74.98 \mathrm{pg} / \mathrm{mL}$, followed by CRP values higher than $81 \mathrm{mg} / \mathrm{L}$, PCT values equal to or higher than $0.56 \mathrm{ng} / \mathrm{mL}$, and D-dimer values equal to or higher than $760 \mathrm{ng} / \mathrm{mL}$ FEU. However, 5 out of 318 participants with IL-6, CRP, PCT, and D-dimer values above cutoff value survived. These findings suggest a good prediction of in-hospital mortality in patients with COVID-19 who require admission to the ICU, especially when jointly using all four cutoff values.

The cytokine storm is one of the most critical factors contributing to COVID-19 mortality. Elevated values of various cytokines, such as IL-1, IL-2, IL-6, IL-7, IL-8, IL-12, IFN, MCP-1, and TNF- $\alpha$, were observed. In SARS-CoV-2positive patients, cytokine storm is characterized by high serum concentrations of IL- 6 and TNF- $\alpha$ predominantly $[19,20]$. Our study observed higher mortality in patients with IL-6 concentrations higher than $74.98 \mathrm{pg} / \mathrm{mL}$. In addition, other studies also favoured a more severe form of the disease and higher mortality of patients with higher values of IL-6 [21-23]. Patients whose maximum IL-6 values exceeded $80 \mathrm{pg} / \mathrm{mL}$ had a significantly higher probability of need of invasive mechanical ventilation. Hyperinflammatory response in the setting of COVID-19 could also be responsible for the potential multiorgan failure and various lifethreatening complications, including ARDS, myocardial damage, and kidney and liver failure. Also, a significant predictor was elevated values of CRP [24].

In addition to IL-6, CRP is a significant marker of COVID-19 inflammation. Higher levels of serum CRP are associated with higher mortality in people with severe COVID-19 disease [25], more specifically, CRP values above $77.35 \mathrm{mg} / \mathrm{L}$ [26]. On the other hand, the CRP threshold, which was found as a predictor of in-hospital mortality by Du et al., was lower, and it was $10 \mathrm{mg} / \mathrm{L}$ [27]. Wang confirmed a positive correlation between CRP values and CT findings in the lungs in the initial stages of the disease. Their findings could give grounds for the connection between high CRP values and a more severe form of the disease [28]. Furthermore, CRP had a significantly better effect in predicting death than age, neutrophil count, and platelet count [29].

Therefore, it is essential to recognize the hyperinflammatory syndrome in COVID-19 patients, primarily over the previous quoted inflammatory parameters, and apply the anti-inflammatory therapy right on time. Corticosteroids, as anti-inflammatory drugs, have shown significant positive effects in patients with a hyperinflammatory response to SARS-CoV2 by reducing mortality, decreasing hospital stay, and increasing ventilator-free days [30, 31]. The antiinflammatory effects of corticosteroids are proven by inducing the synthesis of anti-inflammatory proteins and, on the other hand, by inhibiting the synthesis of proinflammatory proteins [32]. It is crucial to start corticosteroid treatment at the right time and in the right patient since early administration and administration to patients with asymptomatic and milder forms of the disease may have adverse effects [33]. 
TABLE 1: Age, comorbidities, laboratory parameters, and CT score of patients participated in the study. Results are expressed in $n$ (\%) and median (IQR).

\begin{tabular}{|c|c|c|c|c|}
\hline & Total $(n=318)$ & No survivor $(n=195)$ & Survivor $(n=123)$ & $p$ value \\
\hline Age (years) & $69(60-77)$ & $72(64-79)$ & $63(51-73)$ & $<0.001$ \\
\hline Males, $n(\%)$ & $219(68.9)$ & $130(59.4)$ & $89(40.6)$ & \multirow{2}{*}{0.286} \\
\hline Females, $n(\%)$ & $99(31.1)$ & $65(65.7)$ & $34(34.3)$ & \\
\hline \multicolumn{5}{|l|}{ Comorbidities } \\
\hline Hypertension, $n(\%)$ & $223(70.1)$ & $140(71.8)$ & $83(67.5)$ & \\
\hline Diabetes mellitus, $n$ (\%) & $100(31.4)$ & $54(27.7)$ & $46(37.4)$ & \\
\hline Coronary disease, $n(\%)$ & $62(19.5)$ & $37(19)$ & $25(20.3)$ & \\
\hline Obesity, $n(\%)$ & $40(12.6)$ & $21(10.8)$ & $19(15.4)$ & \\
\hline Cardiomyopathy, $n$ (\%) & $27(8.5)$ & $18(9.2)$ & $9(7.3)$ & \\
\hline COPD, $n(\%)$ & $19(6)$ & $13(6.7)$ & $6(4.9)$ & \\
\hline Asthma, $n(\%)$ & $14(4.4)$ & $9(4.6)$ & $5(4.1)$ & \\
\hline \multicolumn{5}{|l|}{ Laboratory parameters } \\
\hline IL-6 (pg/L) & $110.8(44.1-399.6)$ & $160.7(71.4-812.3)$ & $66.8(29.7-239)$ & $<0.001$ \\
\hline CRP (mg/L) & $88(53.8-191.5)$ & $103.4(61.1-210.1)$ & $75.5(41.7-177.2)$ & $<0.001$ \\
\hline Lymphocyte (\%) & $0.7(0.5-1.1)$ & $0.7(0.5-1)$ & $0.8(0.5-1.2)$ & 0.063 \\
\hline Serum ferritin $(\mu \mathrm{g} / \mathrm{L})$ & $822(415.5-1478)$ & 766.5 (374-1409.2) & $760.5(306.7-1416)$ & 0.673 \\
\hline PCT (ng/mL) & $1.1(0.2-9)$ & $3.27(0.8-17.9)$ & $0.2(0.1-0.7)$ & $<0.001$ \\
\hline D-dimer (ng/mL) & $829(497-2759.5)$ & $1121.5(594-3212.2)$ & $666(353.5-1317)$ & $<0.001$ \\
\hline Platelet count $\left(\times 10^{9} / \mathrm{L}\right)$ & $225(161.5-303)$ & $204.5(146-281)$ & $234(179.7-339.5)$ & 0.022 \\
\hline INR & $1.1(1-1.3)$ & $1.1(1-1.3)$ & $1.1(1-1.2)$ & 0.341 \\
\hline $\operatorname{aPTT}(s)$ & $25.6(22.5-30.3)$ & $26(22.7-30.3)$ & $24.6(22.4-28.4)$ & 0.073 \\
\hline Fibrinogen $(\mathrm{g} / \mathrm{L})$ & $4.1(3.5-4.9)$ & $4.1(3.5-5)$ & $4.2(3.4-5.1)$ & 0.921 \\
\hline Albumin (g/L) & $32(29-35)$ & $31(27.5-33)$ & $35(32-38)$ & $<0.001$ \\
\hline CT score & $17(5-22)$ & $17(1.5-22)$ & $17(8-22)$ & 0.96 \\
\hline
\end{tabular}

TABle 2: C-statistic of IL-6, CRP, PCT, and D-dimer to predict mortality in patients with COVID-19.

\begin{tabular}{lcc}
\hline Predictor value & $C$-index & 95\% confidence interval \\
\hline IL-6 & 0.64 & $0.57-0.71$ \\
CRP & 0.62 & $0.56-0.69$ \\
PCT & 0.77 & $0.71-0.83$ \\
D-dimer & 0.64 & $0.57-0.7$ \\
\hline
\end{tabular}

Elevated PCT has been detected in individuals treated for COVID-19 disease. PCT equal to or higher than $0.56 \mathrm{ng} / \mathrm{mL}$ is associated with higher mortality. Elevated PCT levels in individuals are primarily caused by bacterial coinfections, showing a good role in detecting bacterial coinfections and consequently initiating an antibiotic therapy [34]. A meta-analysis that analyzed four studies proved that an increase in PCT is associated with a five times higher risk of a more severe COVID-19 presentation (OR, 4.76; 95\% CI, 2.74-8.29) [35]. Furthermore, another meta-analysis, which included over 10 thousand patients, indicated the importance of elevated PCT values as a predictor of fatal disease outcomes. The same study showed that lymphopenia, thrombocytopenia, elevated D-dimer, elevated CRP, then elevated CK, AST, ALT, LDH, and creatinine are independent predictors of deadly disease outcomes [36].
Our study proved that the concentration of D-dimer above $760 \mathrm{ng} / \mathrm{mL}$ FEU, measured on admission to the ICU, was associated with a higher risk of death during hospitalization. High values of D-dimer in COVID-19 patients are associated with local pulmonary thrombosis, which occurs as an immune-hemostatic response to prevent and limit further spread of the virus. The elevated $\mathrm{D}$-dimer values exist due to a breakdown of these microthrombi $[37,38]$. Elevation of $\mathrm{D}$-dimer during the disease carries a higher risk of progression to severe form and mortality [3]. In particular, elevated values of $\mathrm{D}$-dimer and fibrin degradation products and prolonged prothrombin time were measured at admission in the deceased subjects compared to the cured ones [39]. The study by Klok et al. [9] demonstrated that Ddimer values above $1 \mu \mathrm{g} / \mathrm{mL}$, measured on admission to COVID-19 treatment facilities, were associated with an eighteen times higher risk of death. Further, any increase in Ddimer values by $1 \mu \mathrm{g} / \mathrm{mL}$ on admission is associated with an increase in the risk of death by $6 \%$, as well as an increase in the probability $(8 \%)$ of treatment with mechanical ventilation [40]. Creel-Bulos et al. showed a variation in Ddimer values in the first twenty-five days of hospitalization [41]. There is an almost linear trend of D-dimer increase in the first ten days of treatment, after which D-dimer levels are flattened. Moreover, a steeper D-dimer growth curve was observed in individuals with detected deep vein thrombosis 


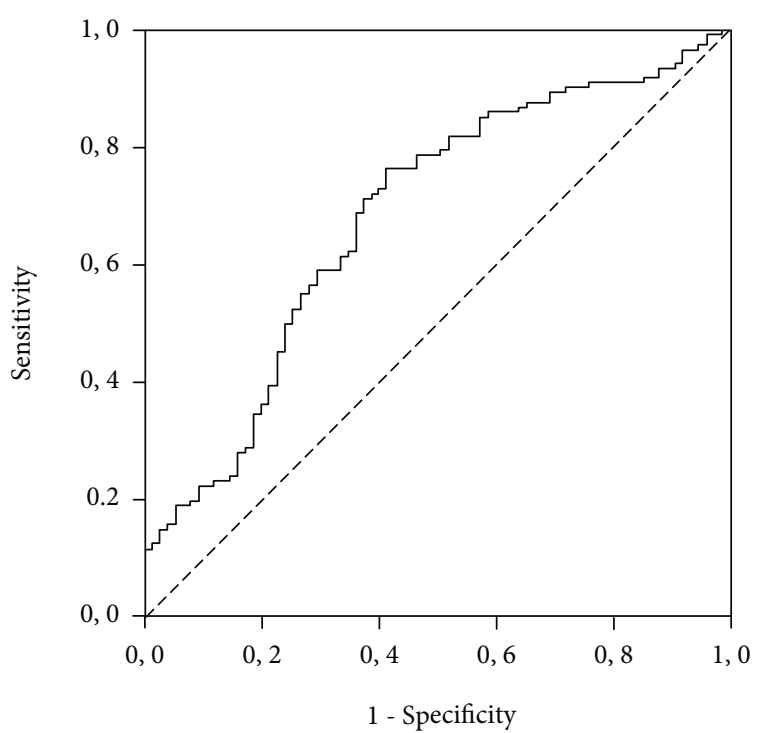

(a)

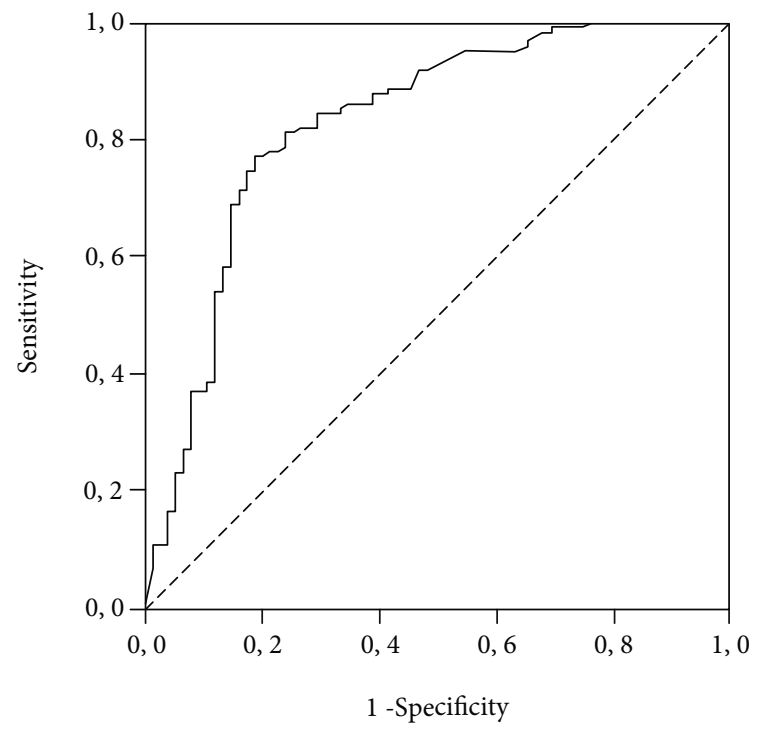

(c)

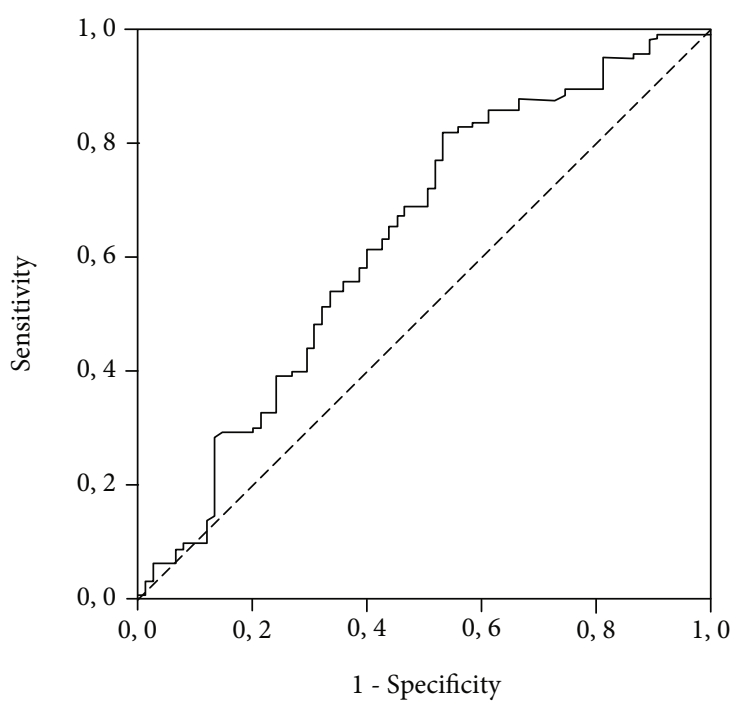

(b)

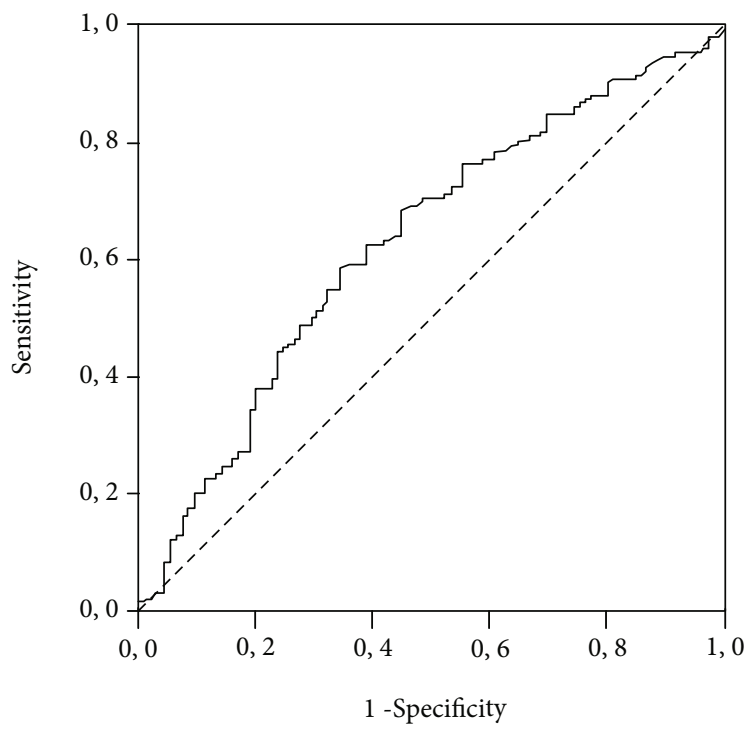

(d)

FIgURE 1: Receiver operator characteristic curve for (a) IL-6, (b) CRP, (c) PCT, and (d) D-dimer to predict deaths. The optimum cutoff point, identified as the point closest to the upper left corner, was for IL-6 (74.98 pg/mL), CRP $(81 \mathrm{mg} / \mathrm{L}), \mathrm{PCT}(0.56 \mathrm{ng} / \mathrm{mL})$, and Ddimer $(760 \mathrm{ng} / \mathrm{mL}$ FEU).

during treatment. In contrast, differences in D-dimer growth curves were not seen in the deceased and those discharged from treatment. Zhang et al. [42] concluded that a Ddimer higher than $2 \mu \mathrm{g} / \mathrm{mL}$ on admission could be considered a predictor of mortality during hospitalization. On the other hand, the study by Soni et al. did not prove that the values of the same parameter above $2 \mu \mathrm{g} / \mathrm{mL}$ measured at admission were mortality predictors [43]. Still, it demonstrated that D-dimer higher than $2 \mu \mathrm{g} / \mathrm{mL}$ during hospitalization is a mortality predictor if the highest measured values are viewed.

The literature has scarce data regarding IL-6, CRP, PCT, and D-dimer values variance in COVID-19 pneumonia and
non-COVID pneumonia. Currently, the best-compared variance of mentioned parameters is between patients with COVID-19 and patients with influenza. Therefore, significantly higher CRP values on hospital admission were detected in influenza-positive subjects after comparing those groups of patients [44]. In their study, Kuang et al. have evinced the higher incidence of influenza patients detected on admission with CRP value above $10 \mathrm{mg} / \mathrm{dl}$ and PCT value above $0.5 \mathrm{ng} / \mathrm{mL}$, compared to COVID-19 patients [45]. On the other hand, a significant increase in IL-6 level was observed in COVID-19 patients compared to patients with influenza [46]. Values of D-dimer were high on admission in both groups, COVID-19 and influenza [47]. During the 


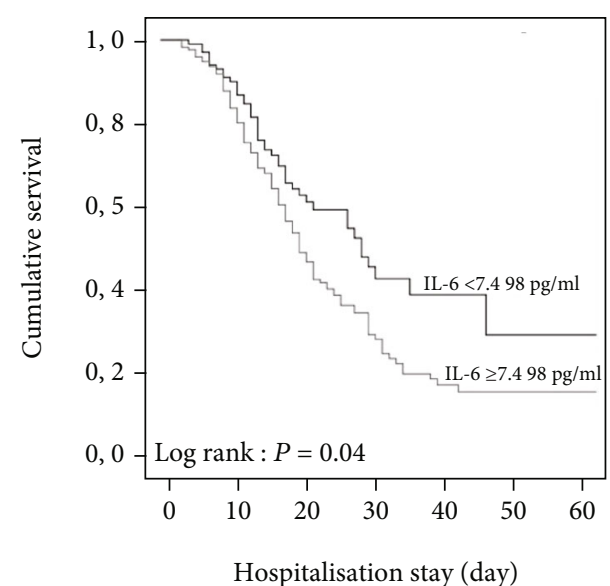

(a)

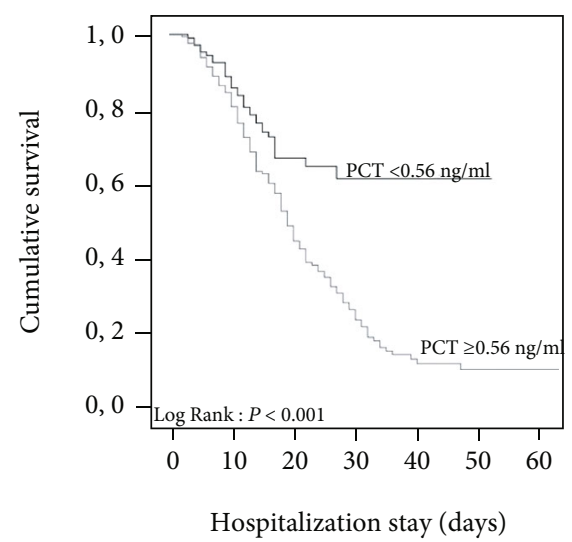

(c)

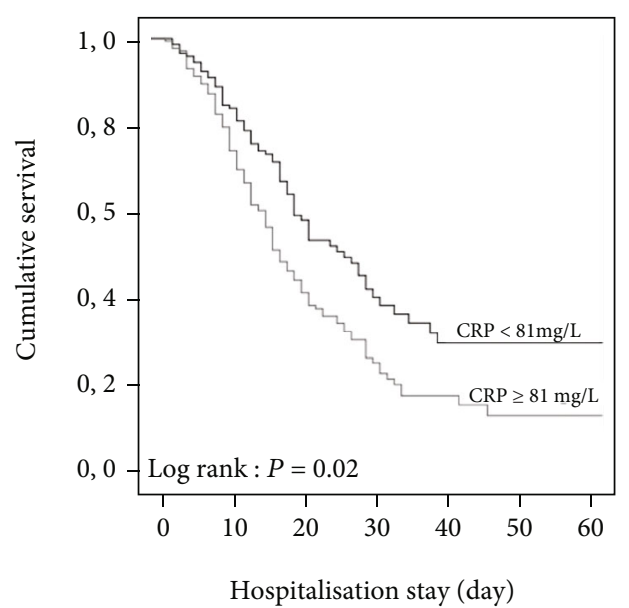

(b)

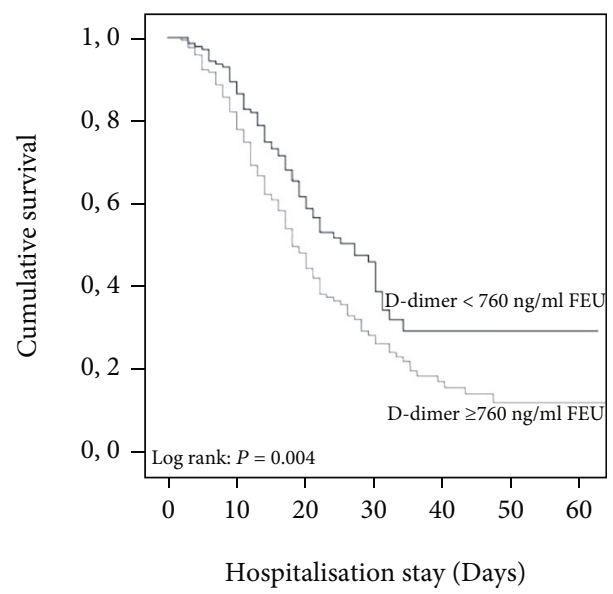

(d)

Figure 2: Kaplan-Meier survival curves for (a) IL-6, (b) CRP, (c) PCT, and (d) D-dimer levels on admission to the ICU.

14-day monitoring of $\mathrm{D}$-dimer values, a significant increase was detected in COVID-19 patients in comparison to influenza patients [48]. Considering everything, COVID-19 and influenza present a potentially life-threatening disease, and therefore, both should be treated with caution. D-dimer dynamics measurement may be used to distinguish COVID-19 infection and influenza infection.

The impact of gender on survival is still debated. Males have a higher chance for severe pneumonia, and therefore, they have a greater chance to be admitted to the ICU [49]. Potential gender-specific mechanisms modulating the course of the disease Gebhard et al. explain with a hormoneregulated expression of genes encoding for the SARS-CoV2 entry receptors ACE 2 receptor and TMPRSS2 as well as sex hormone-driven innate and adaptive immune responses and immunoaging [50]. They stressed out also elucidating the impact of gender-specific lifestyle, health behaviour, psychological stress, and socioeconomic conditions on COVID19 [50]. Our study included 318 patients, $68.9 \%$ male and $31.1 \%$ female, admitted to ICU. These findings are in obedience to the previous study. On the contrary, we did not find a statistically significant difference between genders regarding in-hospital mortality; this statement is supported by the result in Zhou et al.'s study [11]. This can imply that biochemical parameters on admission to the ICU as predictors of in-hospital mortality should be used in all patients with the same prognostic value.

This study has several limitations that should be addressed. First, it is a single-center, retrospective study. The sample size is relatively small. Therefore, the study has limited power to detect the difference between groups. Selection bias is also presented due to the exclusion of patients without D-dimer level on admission to the ICU. Another limitation is the lack of inclusion of some data in the study, such as partial pressure of $\mathrm{O}_{2}, \mathrm{CO}_{2}$, BMI, etc. The reason for this is their absence in the health information system. Furthermore, we did not perform dynamic D-dimer, CRP, IL-6, and PCT measurements because of the study's retrospective design. Incorporating these data might disclose more information and give more power to our study. Unmeasured confounders such as therapy delay, previous corticosteroids use, and BMI could give us residual 
confounding. Finally, performing a multiple-parameter prediction model including D-dimer, CRP, IL-6, and PCT could better predict in-hospital mortality.

\section{Conclusion}

This study supported a growing body of literature regarding hyperinflammatory syndrome and diffuse microvascular thrombosis as predictors of poor clinical outcomes in COVID-19 patients. Proper and on-time differentiation patients with lower survival chances may be crucial for starting anti-inflammatory therapy such as corticosteroids, which may reduce in-hospital mortality. In particular, IL$6 \geq 74.98 \mathrm{pg} / \mathrm{mL}, \quad \mathrm{CRP} \geq 81 \mathrm{mg} / \mathrm{L}, \quad$ PCT $\geq 0.56 \mathrm{ng} / \mathrm{mL}$, and D-dimer $\geq 760 \mathrm{ng} / \mathrm{mL}$ on admission to the ICU could effectively predict in-hospital mortality in COVID-19 patients. Using these laboratory parameters single or in combination may help identify patients with lower survival chances and, on time, improve further treatment. Further prospective multicenter studies are necessary to confirm our findings.

\section{Abbreviations}

SARS-CoV-2:

COVID-19:

ARDS:

DIC:

MODS:

LMWH:

ICU:

IL-6:

CRP:

PCT:

CT:

RT-PCR:

BMI:

COPD:

PT:

aPTT:

IQR:

Sn:

Sp:

IL-1, IL-2, IL-7, IL-8, Interleukins 1, 2, 7, 8, and 12 and IL-12:

IFN:

MCP-1:

TNF- $\alpha$ :

CK:

AST:

ALT:

LDH:

ACE 2:

TMPRSS2:
Severe acute respiratory syndrome coronavirus 2

Coronavirus disease 2019

Acute respiratory distress syndrome

Disseminated intravascular

coagulation

Multiorgan dysfunction syndrome

Low molecular weight heparin

Intensive Care Unit

Interleukin 6

C-reactive protein

Procalcitonin

Computed tomography

Reverse transcription-polymerase

chain reaction

Body mass index

Chronic obstructive pulmonary

disease

Prothrombin time

Activated partial thromboplastin

time

Interquartile range

Sensitivity

Specificity

Interferon

Membrane cofactor protein 1

Tumor necrosis factor $\alpha$

Creatine kinase

Alanine Aminotransferase

Lactate dehydrogenase

Angiotensin converting enzyme 2

Transmembrane Serine Protease 2
Aspartate Aminotransferase

\section{Data Availability}

The data used to support the findings of this study are available from the corresponding author $(\mathrm{AH})$ upon request.

\section{Conflicts of Interest}

The authors declare that there is no conflict of interest regarding the publication of this paper.

\section{Acknowledgments}

The authors would like to acknowledge all healthcare professionals in the first line against COVID-19 disease in the Republic of Serbia and worldwide. Collaborator details are as follows: Tatjana Ivankovic ${ }^{4} \mathrm{MD}$; Anica Divac ${ }^{4} \mathrm{MD}$; Marija Aleksic ${ }^{1} \mathrm{MD}$; Marija Galo ${ }^{1} \mathrm{MD}$; Milica Zejak ${ }^{4} \mathrm{MD}$; Lalatovic Jovana ${ }^{4} \mathrm{MD}$; Maja Popovic ${ }^{4} \mathrm{MD}$; Filip Lukic ${ }^{4}$ MD; Jasna Gacic ${ }^{4} \mathrm{MD}, \mathrm{PhD}$; Natasa Colakovic ${ }^{2,4} \mathrm{MD}$, $\mathrm{PhD}$; Sladjan Timotijevic ${ }^{4} \mathrm{MD}$; Ana Sekulic ${ }^{2,4} \mathrm{MD}, \mathrm{PhD}$; Masa Bojanic ${ }^{4} \mathrm{MD}$; Milica Aleksic ${ }^{4} \mathrm{MD}$; Andrea Manojlovic ${ }^{4}$ $\mathrm{MD}$; Vladan Mudrenovic ${ }^{4} \mathrm{MD}$; Igor Nadj ${ }^{4} \mathrm{MD}$; Stefan Guslarevic ${ }^{4} \mathrm{MD}$; Barbara Loboda ${ }^{4} \mathrm{MD}$; Zoran Andric ${ }^{4} \mathrm{MD}$, $\mathrm{PhD}$; Natasa Petrovic-Stanojevic ${ }^{5,6} \mathrm{MD}, \mathrm{PhD}$; Milica Marjanovic-Petkovic ${ }^{2,5} \mathrm{MD}, \mathrm{PhD}$; Ivan Rovic ${ }^{2}$; Biljana Rakic ${ }^{2}$; Maksim Sarcevic ${ }^{2}$; Milica Minić ${ }^{2}$. ${ }^{1}$ University Clinical centre of Serbia, Belgrade, Serbia. ${ }^{2}$ Faculty of Medicine, University of Belgrade, Belgrade, Serbia. ${ }^{3}$ Blood Transfusion Institute of Serbia, Belgrade, Serbia. ${ }^{4}$ University Clinical Hospital Center Bezanijska Kosa, Belgrade, Serbia. ${ }^{5}$ University Clinical Center Zvezdara, Belgrade, Serbia. ${ }^{6}$ Faculty of stomatology, University of Belgrade, Belgrade, Serbia.

\section{References}

[1] N. Kirtipal, S. Bharadwaj, and S. G. Kang, "From SARS to SARS-CoV-2, insights on structure, pathogenicity and immunity aspects of pandemic human coronaviruses," Infection, Genetics and Evolution, vol. 85, article 104502, 2020.

[2] G. White-Dzuro, L. E. Gibson, L. Zazzeron et al., "Multisystem effects of COVID-19: a concise review for practitioners," Postgraduate Medicine, vol. 133, no. 1, pp. 20-27, 2021.

[3] F. Yang, T. Ning, L. Hefei, and C. Wenjing, "Coagulation Dysfunction," Archives of Pathology \& Laboratory Medicine, vol. 144, no. 10, pp. 1223-1229, 2020.

[4] W. J. Wiersinga, A. Rhodes, A. C. Cheng, S. J. Peacock, and H. C. Prescott, "Pathophysiology, transmission, diagnosis, and treatment of coronavirus disease 2019 (COVID-19): a review," Journal of the American Medical Association, vol. 324, no. 8, pp. 782-793, 2020.

[5] H. M. Zawbaa, H. Osama, A. El-Gendy et al., "Effect of mutation and vaccination on spread, severity, and mortality of COVID-19 disease," Journal of Medical Virology, vol. 94, no. 1, pp. 197-204, 2022.

[6] S. Middeldorp, M. Coppens, T. F. van Haaps et al., "Incidence of venous thromboembolism in hospitalized patients with COVID-19," Journal of Thrombosis and Haemostasis, vol. 18, no. 8, pp. 1995-2002, 2020.

[7] S. Cui, S. Chen, X. Li, S. Liu, and F. Wang, "Prevalence of venous thromboembolism in patients with severe novel 
coronavirus pneumonia," Journal of Thrombosis and Haemostasis, vol. 18, no. 6, pp. 1421-1424, 2020.

[8] A. Porfidia, E. Valeriani, R. Pola, E. Porreca, A. W. S. Rutjes, and M. Di Nisio, "Venous thromboembolism in patients with COVID-19: systematic review and meta-analysis," Thrombosis Research, vol. 196, pp. 67-74, 2020.

[9] F. A. Klok, M. J. Kruip, N. J. Van der Meer et al., "Incidence of thrombotic complications in critically ill ICU patients with COVID-19," Thrombosis Research, vol. 191, pp. 145-147, 2020.

[10] C. Huang, Y. Wang, X. Li et al., "Clinical features of patients infected with 2019 novel coronavirus in Wuhan, China," Lancet, vol. 395, no. 10223, pp. 497-506, 2020.

[11] F. Zhou, T. Yu, R. Du et al., "Clinical course and risk factors for mortality of adult inpatients with COVID-19 in Wuhan, China: a retrospective cohort study," Lancet, vol. 395, no. 10229, pp. 1054-1062, 2020.

[12] W. J. Guan, Z. Y. Ni, Y. Hu et al., "Clinical characteristics of coronavirus disease 2019 in China," The New England Journal of Medicine, vol. 382, no. 18, pp. 1708-1720, 2020.

[13] H. H. Yu, C. Qin, M. Chen, W. Wang, and D. S. Tian, "Ddimer level is associated with the severity of COVID-19," Thrombosis Research, vol. 195, pp. 219-225, 2020.

[14] J. Lin, H. Yan, H. Chen et al., "COVID-19 and coagulation dysfunction in adults: a systematic review and meta-analysis," Journal of Medical Virology, vol. 93, no. 2, pp. 934-944, 2021.

[15] S. Kiss, N. Gede, P. Hegyi et al., "Early changes in laboratory parameters are predictors of mortality and ICU admission in patients with COVID-19: a systematic review and meta-analysis," Medical Microbiology and Immunology, vol. 210, no. 1, pp. 33-47, 2021.

[16] A. D. Force, V. M. Ranieri, G. Rubenfeld et al., "Acute respiratory distress syndrome: the Berlin Definition," Journal of the American Medical Association, vol. 307, no. 23, pp. 25262533, 2012.

[17] M. Pelemiš, G. Stevanović, V. Turkulov et al., National Protocol of the Republic of Serbia to Treat COVID-19 Infection, 10th edition10th edition, , 2020.

[18] World Health Organisation, COVID-19 clinical management: living guideline, WHO, Geneva, 2021.

[19] Y. Tang, J. Liu, D. Zhang, Z. Xu, J. Ji, and C. Wen, “Cytokine storm in COVID-19: the current evidence and treatment strategies," Frontiers in Immunology, vol. 11, p. 1708, 2020.

[20] B. Hu, S. Huang, and L. Yin, "The cytokine storm and COVID19," Journal of Medical Virology, vol. 93, pp. 250-256, 2021.

[21] M. Tan, Y. Liu, R. Zhou et al., "Immunopathological characteristics of coronavirus disease 2019 cases in Guangzhou, China," Immunology, vol. 160, no. 3, pp. 261-268, 2020.

[22] J. M. Galván-Román, S. C. Rodríguez-García, E. Roy-Vallejo et al., "IL-6 serum levels predict severity and response to tocilizumab in COVID-19: an observational study," Journal of Allergy and Clinical Immunology, vol. 147, no. 1, pp. 7280.e8, 2021.

[23] M. Luo, J. Liu, W. Jiang, S. Yue, H. Liu, and S. Wei, "IL-6 and CD8+ T cell counts combined are an early predictor of inhospital mortality of patients with COVID-19," JCI Insight, vol. 5, no. 13, article e139024, 2020.

[24] T. Herold, V. Jurinovic, C. Arnreich et al., "Elevated levels of IL- 6 and CRP predict the need for mechanical ventilation in COVID-19," Journal of Allergy and Clinical Immunology, vol. 146, no. 1, pp. 128-136.e4, 2020.
[25] J. J. Zhang, Y. Y. Cao, G. Tan et al., "Clinical, radiological, and laboratory characteristics and risk factors for severity and mortality of 289 hospitalized COVID-19 patients," Allergy, vol. 76, no. 2, pp. 533-550, 2021.

[26] F. Pan, L. Yang, Y. Li et al., "Factors associated with death outcome in patients with severe coronavirus disease-19 (COVID19): a case-control study," International Journal of Medical Sciences, vol. 17, no. 9, pp. 1281-1292, 2020.

[27] L. L. R. Du RH, C. Q. Yang, W. Wang et al., "Predictors of mortality for patients with COVID-19 pneumonia caused by SARS-CoV-2," European Respiratory Journal, vol. 56, no. 3, p. 2002961, 2020.

[28] L. Wang, "C-reactive protein levels in the early stage of COVID-19," Médecine et Maladies Infectieuses, vol. 50, no. 4, pp. 332-334, 2020.

[29] X. Luo, W. Zhou, X. Yan et al., "Prognostic value of C-reactive protein in patients with coronavirus 2019," Clinical Infectious Diseases, vol. 71, no. 16, pp. 2174-2179, 2020.

[30] RECOVERY Collaborative Group, "Dexamethasone in hospitalized patients with Covid-19," The New England Journal of Medicine, vol. 384, no. 8, pp. 693-704, 2021.

[31] WHO Rapid Evidence Appraisal for COVID-19 Therapies (REACT) Working Group, "Association Between Administration of Systemic Corticosteroids and Mortality Among Critically Ill Patients With COVID-19: A Meta-analysis," JAMA, vol. 324, no. 13, pp. 1330-1341, 2020.

[32] D. Annane, “Corticosteroids for COVID-19," Journal of Intensive Medicine, vol. 1, no. 1, pp. 14-25, 2021.

[33] M. Johns, S. George, M. Taburyanskaya, and Y. K. Poon, "A review of the evidence for corticosteroids in COVID-19," Journal of Pharmacy Practice, vol. 15, p. 089719002199850 , 2021.

[34] P. Williams, C. McWilliams, K. Soomro et al., "The dynamics of procalcitonin in COVID-19 patients admitted to intensive care unit - a multi-centre cohort study in the South West of England, UK," Journal of Infection, vol. 82, no. 6, pp. e24e26, 2021.

[35] G. Lippi and M. Plebani, "Procalcitonin in patients with severe coronavirus disease 2019 (COVID-19): a meta-analysis," Clinica chimica acta; international journal of clinical chemistry, vol. 505, pp. 190-191, 2020.

[36] P. Malik, U. Patel, D. Mehta et al., "Biomarkers and outcomes of COVID-19 hospitalisations: systematic review and metaanalysis," BMJ evidence-based medicine, vol. 26, no. 3, pp. 107-108, 2021.

[37] J. Thachil and A. Srivastava, "SARS-2 coronavirus-associated hemostatic lung abnormality in COVID-19: is it pulmonary thrombosis or pulmonary embolism?," Seminars in Thrombosis and Hemostasis, vol. 46, pp. 777-780, 2020.

[38] T. Iba, J. H. Levy, M. Levi, J. M. Connors, and J. Thachil, "Coagulopathy of coronavirus disease 2019," Critical Care Medicine, vol. 48, no. 9, pp. 1358-1364, 2020.

[39] N. Tang, D. Li, X. Wang, and Z. Sun, “Abnormal coagulation parameters are associated with poor prognosis in patients with novel coronavirus pneumonia," Journal of thrombosis and haemostasis, vol. 18, no. 4, pp. 844-847, 2020.

[40] L. Naymagon, N. Zubizarreta, J. Feld et al., "Admission Ddimer levels, D-dimer trends, and outcomes in COVID-19," Thrombosis Research, vol. 196, pp. 99-105, 2020.

[41] C. Creel-Bulos, M. Liu, S. C. Auld et al., "Trends and diagnostic value of $\mathrm{D}$-dimer levels in patients hospitalized with 
coronavirus disease 2019," Medicine (Baltimore), vol. 99, no. 46, article e23186, 2020.

[42] L. Zhang, X. Yan, Q. Fan et al., "D-dimer levels on admission to predict in-hospital mortality in patients with Covid-19," Journal of thrombosis and haemostasis, vol. 18, no. 6, pp. 1324-1329, 2020.

[43] M. Soni, R. Gopalakrishnan, R. Vaishya, and P. Prabu, "Ddimer level is a useful predictor for mortality in patients with COVID-19: analysis of 483 cases," Diabetes \& Metabolic Syndrome: Clinical Research \& Reviews, vol. 14, no. 6, pp. 22452249, 2020.

[44] J. Li, S. Li, X. Qiu, W. Zhu, L. Li, and B. Qin, "Performance of diagnostic model for differentiating between COVID-19 and influenza: a 2-center retrospective study," Medical Science Monitor, vol. 27, article e932361, 2021.

[45] P. D. Kuang, C. Wang, H. P. Zheng et al., "Comparison of the clinical and CT features between COVID-19 and H1N1 influenza pneumonia patients in Zhejiang, China," European Review for Medical and Pharmacological Sciences, vol. 25, no. 2, pp. 1135-1145, 2021.

[46] A. H. Karaba, W. Zhou, L. L. Hsieh et al., "Differential cytokine signatures of SARS-CoV-2 and influenza infection highlight key differences in pathobiology," Clinical Infectious Diseases, vol. 74, no. article ciab376, 2021.

[47] J. Zhang, X. Huang, D. Ding, and Z. Tao, "Platelet-driven coagulopathy in COVID-19 patients: in comparison to seasonal influenza cases," Experimental Hematology \& Oncology, vol. 10, no. 1, p. 34, 2021.

[48] Y. Mei, S. E. Weinberg, L. Zhao et al., "Risk stratification of hospitalized COVID-19 patients through comparative studies of laboratory results with influenza," EClinicalMedicine, vol. 26, article 100475, 2020.

[49] P. Mo, Y. Xing, Y. U. Xiao et al., "Clinical characteristics of refractory COVID-19 pneumonia in Wuhan, China," Clinical infectious diseases, vol. 16, p. 270, 2020.

[50] C. Gebhard, V. Regitz-Zagrosek, H. K. Neuhauser, R. Morgan, and S. L. Klein, "Impact of sex and gender on COVID-19 outcomes in Europe," Biology of Sex Differences, vol. 11, no. 1, p. 29, 2020. 